\title{
Nivolumab, a Double-Edged Sword: A Case Report of Nivolumab-Induced Myasthenia Gravis
}

\author{
Nayha Tahir ${ }^{\mathrm{a}, \mathrm{e}}$, Anber Mahboob ${ }^{\mathrm{b}}$, Xuanzhen Piao $^{\mathrm{c}}$, Grace Ying ${ }^{\mathrm{a}}$, Jishna Shrestha ${ }^{\mathrm{a}}$, \\ Robin Sherchan ${ }^{\mathrm{a}}$, Farha Zahra ${ }^{\mathrm{d}}$
}

\begin{abstract}
Nivolumab is a checkpoint inhibitor approved to treat various solid organs malignancies. Although checkpoint inhibitors are very efficacious, these medications are also associated with a variety of side effects that could be life-threatening. We present a case of nivolumab-induced myasthenia gravis in a patient with stage IV esophageal cancer, who was found to have generalized weakness, blurry vision, diplopia, and later developed acute hypoxic respiratory failure with subsequent intubation. The patient was treated with intravenous immunoglobulin and plasmapheresis, and later started on pyridostigmine and high-dose steroids with minimal improvement. Goals of care were discussed with the patient and family, and the decision was made to discharge the patient home with hospice care. Nivolumab-induced myasthenia gravis is very aggressive with a poor prognosis if not appropriately managed in time. Hence we strongly recommend a multidisciplinary approach, including neurologists, to monitor patients on nivolumab therapy to reduce morbidity and mortality associated with it.
\end{abstract}

Keywords: Nivolumab; Myasthenia gravis; Esophageal cancer; PDL1 inhibitors

\section{Introduction}

Nivolumab is a monoclonal antibody belonging to the checkpoint inhibitors, recently approved to treat recurrent or meta-

Manuscript submitted September 3, 2021, accepted September 22, 2021

Published online September 29, 2021

${ }^{a}$ Chicago Medical School, Rosalind Franklin University of Medicine and Science, Internal Medicine Residency Program at Northwestern Medicine Hospital, McHenry, IL, USA

bSharif Medical and Dental College, Lahore, Pakistan

${ }^{\mathrm{c}}$ Chicago Medical School, Rosalind Franklin University of Medicine and Science, North Chicago, IL, USA

dDepartment of Internal Medicine, Northwestern Medicine McHenry Hospital, McHenry, IL, USA

${ }^{\mathrm{e}}$ Corresponding Author: Nayha Tahir, Chicago Medical School, Rosalind Franklin University of Medicine and Science, Internal Medicine Residency Program at Northwestern Medicine Hospital, McHenry, IL, USA.

Email: nayha.tahir@rosalindfranklin.edu

doi: https://doi.org/10.14740/jmc3783 static non-small cell lung cancer, melanoma, renal cell carcinoma, and advanced esophageal carcinoma [1]. The use of checkpoint inhibitors against various molecules, including programmed cell death protein-1 (PD-1), programmed death ligand-1 (PD-L1), and cytotoxic T-lymphocyte-associated protein-4 (CTLA-4), has become widespread in clinical practice [1]. However, although very efficacious, these medications can induce various adverse effects, the full spectrum of which is not yet thoroughly characterized.

This case highlights a case of PD-1 inhibitor-induced myasthenia gravis $(\mathrm{MG})$ in a patient with stage IV esophageal cancer: a severe adverse event that has been increasingly observed with the use of PD-1/PD-L1 inhibitors.

\section{Case Report}

\section{Investigations}

A 76-year-old man presented to the emergency department after a mechanical fall at home with head trauma. He attempted to get up from a chair using a cane and fell to the floor. His past medical history includes metastatic esophageal adenocarcinoma status post chemotherapy and radiation, radiation-induced esophageal stricture status post nasogastric tube placement, bilateral malignant pleural effusion, complete heart block status post dual-chamber pacemaker placement 1 month before presentation, recent non-ST segment elevation myocardial infarction with a normal nuclear stress test, chronic diastolic heart failure with a preserved ejection fraction of $79 \%$, chronic normocytic anemia with baseline hemoglobin of $7.7-8.9 \mathrm{~g} /$ $\mathrm{dL}$, type 2 diabetes mellitus, hypertension, hyperlipidemia, and history of prostate cancer status post radiation. The patient stated that he was also having trouble keeping his eyes open for 2 weeks before presentation, so he underwent an outpatient magnetic resonance imaging (MRI) of the brain with and without contrast, which was negative for intracranial metastasis or hemorrhage. The patient's wife denied observing seizure activities, urinary or fecal incontinence, and slurred speech, and stated that the patient was likely unresponsive for a few seconds. Patient denied any prodromal symptoms such as chest pain, palpitation, shortness of breath, headaches, dizziness, nausea, vomiting, weakness, or numbness.

The patient was a former smoker who quit about 35 years ago, with no history of alcohol or any recreational drugs use. 
Table 1. Basic Labs of the Patient

\begin{tabular}{llllll}
\hline Variables & Day $\mathbf{1}$ & Day $\mathbf{5}$ & Day 9 & Day 13 & Day of discharge \\
\hline WBC (NV: $\left.4.1-11.0 \times 10^{3} / \mu \mathrm{L}\right)$ & 10.6 & 5.1 & 9.9 & 5.5 & 6.9 \\
Neutrophils (NV: $\left.2.1-8.4 \times 10^{3} / \mu \mathrm{L}\right)$ & 8.8 & 4.4 & 8.4 & 4.2 & 5.1 \\
Hemoglobin $(\mathrm{NV}: 12.0-16.0 \mathrm{~g} / \mathrm{dL})$ & 8.0 & 7.7 & 7.5 & 8.6 & 11.2 \\
Platelet $\left(\mathrm{NV}: 150-400 \times 10^{3} / \mu \mathrm{L}\right)$ & 130 & 89 & 82 & 109 & 115 \\
BUN $(\mathrm{NV}: 8-24 \mathrm{mg} / \mathrm{dL})$ & 30 & 25 & 60 & 51 & 48 \\
Creatinine $(\mathrm{NV}: 0.55-1.30 \mathrm{mg} / \mathrm{dL})$ & 1.14 & 0.92 & 0.86 & 0.78 & 0.65 \\
Fibrinogen $(\mathrm{NV}: 200-400 \mathrm{mg} / \mathrm{dL})$ & $\mathrm{N} / \mathrm{A}$ & 165 & 171 & 203 & 199 \\
Arterial blood pH $(\mathrm{NV}: 7.38-7.42)$ & 7.42 & 7.40 & 7.47 & 7.47 & N/A \\
\hline
\end{tabular}

WBC: white blood cell; NV: normal value; BUN: blood urea nitrogen; N/A: not applicable.

Family history was pertinent for ovarian cancer in mother and lung cancer in father. Home medications include aspirin, carvedilol, furosemide, metformin, and rosuvastatin. The patient was started on radiation therapy with concurrent chemotherapy with weekly carboplatin and paclitaxel 6 months before presentation. The patient tolerated chemotherapy for 6 weeks with interruption of treatment secondary to neutropenia and was then initiated on FOLFOX chemotherapy with pegfilgrastim and nivolumab 3 and 2 months before presentation, respectively. Off note, interval reduction of chemotherapy dose took place about 5 weeks ago secondary to thrombocytopenia, and 4 weeks ago chemotherapy was held secondary to generalized weakness and functional deconditioning.

On presentation, patients' blood pressure was 121/69 mm $\mathrm{Hg}$, heart rate 83 beats per minute (bpm), temperature 36.6 ${ }^{\circ} \mathrm{C}$, respiratory rate 13 breaths per minute, and oxygen saturation $96 \%$ on room air. He was alert, awake, and oriented to person, time, place, and situation. On the physical exam, the patient was noted to have mild bibasilar crackles. Additionally, bilateral ptosis was noted. Otherwise, no cranial nerve or focal neurological deficits were noted. The rest of the physical examination was unremarkable.

\section{Diagnosis}

Basic labs are included in Table 1. Electrocardiogram (EKG) showed ventricular-paced rhythm at $87 \mathrm{bpm}$ with no acute ischemic changes. Computed tomography (CT) of the head showed scattered areas of subarachnoid hemorrhage involving the left frontal, right temporal and inferior right frontal lobes without significant mass or midline shift. CT angiogram of the head was negative for significant stenosis or aneurysm in the head or neck arterial vasculature. CT cervical spine without contrast was negative for acute fractures. Per neurosurgery, no surgical intervention was indicated.

\section{Treatment}

The patient was then started on levetiracetam for seizure prophylaxis. The repeat $\mathrm{CT}$ head without contrast demonstrated stable findings.

\section{Follow-up and outcomes}

A few days later, the patient started complaining of worsened blurry vision, fatigue, and diplopia in both extremes of horizontal gaze. On the physical exam, severe bilateral ptosis was noted to the point where the patient could not keep his eyes open without helping with his fingers. In terms of muscle strength, it was showed that neck flexion $3 / 5$, extension $5 / 5$, deltoid $5 / 5$ bilaterally, biceps $4 / 5$ bilaterally, triceps $4 / 5$ bilaterally; hip flexion $2 / 5$ on the right and $3 / 5$ on the left, knee flexion $3 / 5$ on the right and $4 / 5$ on the left, and plantar flexion $4 / 5$ bilaterally. The sensation was intact to light touch, and reflexes were $2+$ throughout. The patient remained awake but somnolent, followed basic commands, and used hand signals to answer questions. Overnight, the patient was noted to have an event of unresponsiveness with oxygen saturation of $80 \%$ on room air, unable to follow commands. Lung examination revealed significant bibasilar crackles with chest radiograph showing bilateral pleural effusions; and the patient was subsequently intubated and transferred to the intensive care unit due to acute hypoxic hypercapnic respiratory failure.

A preliminary diagnosis of MG with ongoing crisis likely secondary to immune checkpoint inhibitor (ICI) therapy (nivolumab) was made, and a diagnostic workup was started. In addition to suspicion of MG, several other paraneoplastic and autoimmune conditions were also included as part of the differential diagnosis. Acetylcholine receptor modulating, binding, blocking antibodies, anti-smooth muscle antibody with titers, and autoimmune comprehensive neurology antibody panels (Table 2) were sent. Lactate dehydrogenase (LDH) was noted to be elevated up to $471 \mathrm{U} / \mathrm{L}$ (reference range: 140 - $280 \mathrm{U} / \mathrm{L}$ ). Aspartate aminotransferase (AST), alanine aminotransferase (ALT), creatinine kinase (CK), thyroid stimulating hormone (TSH), adrenocorticotropic hormone (ACTH), and cortisol levels were checked and were noted to be within normal limit. CT chest without contrast was negative for any findings concerning thymoma but revealed bilateral pleural effusions. Ultrasound-guided thoracentesis was subsequently performed with $950 \mathrm{~mL}$ of slightly cloudy aspirate removal with fluid studies consistent with metastatic disease. Electromyography (EMG) and muscle biopsy was planned by neurology but the patient's wife denied to pursuing any further 
Table 2. Autoimmune Comprehensive Neurology Antibody Panels

\begin{tabular}{ll}
\hline & Results \\
\hline Titin antibody (reference: $<11 \mathrm{SI}$ ) & 91 \\
Striated muscle antibody & Positive \\
Striated muscle antibody titer (reference: $<1: 40)$ & $1: 160$ \\
Acetylcholine receptor modulating antibody (reference: $<32 \%$ inhibition) & $<6 \%$ \\
Acetylcholine receptor blocking antibody (reference: $<15 \%$ inhibition) & $<15 \%$ \\
Acetylcholine receptor binding antibody & $<0.30$ \\
$\quad$ Negative: $\leq 0.30$ nmol/L & \\
$\quad$ Equivocal: $0.31-0.49$ nmol/L & Negative \\
$\quad$ Positive: $\geq 0.50$ nmol/L & \\
Autoimmune comprehensive neurology antibody panel & \\
\hline
\end{tabular}

diagnostic workup. The patient was started on pyridostigmine and high-dose prednisone by neurology service and also completed 4 days of intravenous immunoglobulin (IVIG) and 5 days of plasmapheresis. Post therapy, our patient could semiopen his eyes and be placed on pressure support for a trial of extubation. The patient expressed his wishes to pursue comfort care. Therefore, a hospice consult was placed, and the patient was discharged home with hospice care on pyridostigmine and prednisone.

\section{Discussion}

Advances in cancer treatment over the years have established ICIs such as CTLA-4 and PD-1/PD-L1 blocking agents as a standard of care in several cancers [2]. PD-1 binds to specific ligands (PD-L1 (B7-H1) and PD-L2 (B7-DC)) that are expressed on tumor cells, resulting in selective immunosuppression and prevention of the immune system from rejecting the tumor. Our point of discussion is one particular drug, nivolumab, a monoclonal antibody that blocks PD-1, an immune checkpoint receptor expressed by activated T cells, from binding to its ligands [3]. Even with impressive responses to ICIs in a wide variety of malignancies, these agents cause class-related adverse effects, called immune-related adverse events (irAEs), which possibly result from an imbalanced selfactivation of a T-cell immune response [4]. Immune checkpoints have an essential role in maintaining self-tolerance and warding off autoimmunity. When these physiologic "blocks" on cell-mediated immunity are removed, it can lead to unfavorable effects on a large scale beyond the tumor site. When these unchecked activated $\mathrm{T}$ cells circulate widely targeting self-antigens and inflammatory cytokines, it may result in inflammation and destruction in peripheral tissue, manifesting clinically as an autoimmune disease [5]. Nivolumab-induced irAE can affect all body organs, causing diabetes, hypothyroidism, adrenal insufficiency, interstitial pneumonia, colitis, renal and liver dysfunction, MG, myositis, and many others. Even though MG occurs in a smaller proportion of patients treated with nivolumab than other side effects, it does hold significant importance due to its severity and prognosis. Sev- eral cases have been recorded documenting MG development in patients on nivolumab therapy after one or more cycles. The onset and progression remain unclear, and a systematic approach is needed to establish its true incidence [6]. The exact pathogenesis of nivolumab causing MG is not entirely understood yet, but one hypothesis suggests that it promotes antigen-antibody reaction mediated by $\mathrm{T}$ lymphocytes or promotes the activation of B lymphocytes by T2 lymphocytes. Another hypothesis suggests that it may potentiate the autoimmune response in patients who were previously sensitive against acetylcholine receptors having positive acetylcholine receptor antibody titers but had no clinical manifestations [7]. One cohort study documented that ICI-related MG was more common in the older age group than patients with spontaneous MG. This finding indicates that elderly patients with cancer might be more susceptible to this particular adverse effect [2]. Multiple cases of ICI-associated MG have also been reported with myalgias and increased creatine phosphokinase (CPK) levels without myositis diagnosis, which suggests that the actual incidence of concurrent myositis could be higher [5]. Various studies have shown that more than one-third of such patients have overlapping myositis/myocarditis with $\mathrm{MG}$, and this particular subset of patients have severe symptoms and worse clinical outcomes than patients with isolated idiopathic $\mathrm{MG}$ (iMG) [2]. The data available so far suggest that nivolumab-induced $\mathrm{MG}$ is more aggressive, has a worse prognosis, and has higher mortality than spontaneous $\mathrm{MG}$; however, having older mean age at diagnosis and concurrent advanced-stage tumors may influence the prognosis [8]. Approximately $85 \%$ of patients with spontaneous MG have antibodies to the acetylcholine receptor, but these antibodies are frequently negative in ICI-mediated MG, and when they are detected, titers are mostly much lower than those seen in spontaneous MG [5]. Therefore, it is suggested to set a low threshold for prompt extensive treatment initiation in patients with iMG, particularly patients with overlap syndrome, i.e., neuropathy/myositis/MG [9]. Diagnostic workup should include acetylcholine receptor antibodies, muscle-specific antibodies, cerebrospinal fluid analysis, as many patients with irAEs show unexpectedly high cerebrospinal fluid cell counts. Other diagnostic modalities should be medical imaging or 
electroneurography [9]. Findings that support the diagnosis of MG are: 1) positive blood anti-AChR or anti-MuSK antibodies; 2) positive response to cholinesterase inhibitors; and 3 ) decremental responses to repetitive nerve stimulation or abnormal single fiber EMG [10].

ICI-associated MG is treated by first discontinuing the offending drug and then initiating low- dose corticosteroids with slow titration or methylprednisolone. Plasma exchange (PLEX) or IVIG should be added in severe cases [5]. Previous studies show that patients who received IVIG or PLEX as first-line treatment options experienced better outcomes than those treated with steroids alone. Data also suggest that IVIG or PLEX may be more effective when used as a firstline treatment option as many patients deteriorated despite second-line use of IVIG or PLEX secondary to failed initial treatment with corticosteroids. Provided the acute onset and rapid deterioration, it is recommended to use IVIG or PLEX early in the management of ICI-related MG regardless of the severity of initial symptoms. Another problem with using steroids as the only first-line treatment is their well-known side effect of acute exacerbation of iMG symptoms [2, 11]. Even though this worsening is transient, it occurs in almost $50 \%$ of patients, and it has a high potential of progressing to respiratory compromise.

Additionally, steroids need several weeks to show clinical response [12]. Based on documented improvement in outcomes with IVIG and PLEX in the majority of the patients with severe iMG [13-15], their early use is recommended before or simultaneously with steroids to combat the risk of a transient worsening, especially in severe cases [11, 16, 17]. Based on our experience and previously documented literature, we suggest that a multidisciplinary approach [2] should be implemented while treating patients with ICI so that life-threatening adverse effects could be managed in a time-sensitive manner. The team should specifically include neurologists and neuro-oncologists [18], as neurotoxicity can be challenging to diagnose mainly when these agents are administered and monitored by non-neurological professionals hence the risk of underreporting stays high [19]. A standardized stepwise checklist [19] should also be developed to detect the neurological irAEs early, particularly in those hospitals where neurologists are either not readily available or are not directly involved in the care and monitoring of cancer patients [19].

\section{Conclusions}

Although ICIs' exceptional efficacy has transformed the treatment of many cancers, they come with many potentially lethal immune-related adverse effects affecting multiple organs. Nivolumab-induced $\mathrm{MG}$, developed through an unknown mechanism, is very aggressive with poor prognosis if not appropriately managed in time; hence we strongly recommend a multidisciplinary approach, including neurologists, to monitor patients on nivolumab therapy in order to reduce morbidity and mortality associated with it. An aggressive phase IV surveillance can help understand the pathogenesis and probability of MG occurrence and develop an effective manage- ment strategy to enhance the safe use of an otherwise excellent treatment option.

\section{Acknowledgments}

None to declare.

\section{Financial Disclosure}

No financial disclosure or funding to declare.

\section{Conflict of Interest}

No known conflict of interest to disclose.

\section{Informed Consent}

Not applicable.

\section{Author Contributions}

NT wrote the majority of the manuscript. AM, XP, GY, JS, and $\mathrm{RS}$ all contributed to writing and revising. FZ assisted in revisions of final draft.

\section{Data Availability}

The authors declare that data supporting the findings of this study are available within the article.

\section{References}

1. Rajan A, Kim C, Heery CR, Guha U, Gulley JL. Nivolum$\mathrm{ab}$, anti-programmed death-1 (PD-1) monoclonal antibody immunotherapy: Role in advanced cancers. Hum Vaccin Immunother. 2016;12(9):2219-2231.

2. Safa H, Johnson DH, Trinh VA, Rodgers TE, Lin H, Suarez-Almazor ME, Fa'ak F, et al. Immune checkpoint inhibitor related myasthenia gravis: single center experience and systematic review of the literature. J Immunother Cancer. 2019;7(1):319.

3. Sosman JA. Immunotherapy of advanced melanoma with immune checkpoint inhibition. Literature review current through: Jun 2021. This topic last updated: Mar 04, 2021. In UpToDate: Accessed on July 19th, 2021.

4. Cortellini A, Napoleoni L, Cimini N, Parisi A, Pavese F, D'Orazio C, Verna L, et al. Immune checkpoint inhibitors and myasthenic syndromes: a case report of a metastatic renal cell carcinoma patient treated with nivolumab. J Clin Neuromuscul Dis. 2018;20(2):99-100.

5. Haugh AM, Probasco JC, Johnson DB. Neurologic com- 
plications of immune checkpoint inhibitors. Expert Opin Drug Saf. 2020;19(4):479-488.

6. Nakanishi S, Nishida S, Miyazato M, Goya M, Saito S. A case report of nivolumab-induced myasthenia gravis and myositis in a metastatic renal cell carcinoma patient. Urol Case Rep. 2020;29:101105.

7. Minon-Fernandez B, Losada-Domingo JM, SanchezHorvath MT, Barcena-Llona J. [Myasthenia gravis associated with nivolumab]. Rev Neurol. 2020;70(2):72-73.

8. Makarious D, Horwood K, Coward JIG. Myasthenia gravis: An emerging toxicity of immune checkpoint inhibitors. Eur J Cancer. 2017;82:128-136.

9. Mohn N, Beutel G, Gutzmer R, Ivanyi P, Satzger I, Skripuletz T. Neurological immune related adverse events associated with nivolumab, ipilimumab, and pembrolizum$\mathrm{ab}$ therapy-review of the literature and future outlook. J Clin Med. 2019;8(11):1777.

10. Valenti-Azcarate R, Esparragosa Vazquez I, Toledano Illan C, Idoate Gastearena MA, Gallego Perez-Larraya J. Nivolumab and Ipilimumab-induced myositis and myocarditis mimicking a myasthenia gravis presentation. Neuromuscul Disord. 2020;30(1):67-69.

11. Miller RG, Milner-Brown HS, Mirka A. Prednisone-induced worsening of neuromuscular function in myasthenia gravis. Neurology. 1986;36(5):729-732.

12. Guptill JT, Soni M, Meriggioli MN. Current treatment, emerging translational therapies, and new therapeutic targets for autoimmune myasthenia gravis. Neurotherapeutics. 2016;13(1):118-131.
13. Gajdos P, Chevret S, Clair B, Tranchant C, Chastang C. Clinical trial of plasma exchange and high-dose intravenous immunoglobulin in myasthenia gravis. Myasthenia Gravis Clinical Study Group. Ann Neurol. 1997;41(6):789-796.

14. Barth D, Nabavi Nouri M, Ng E, Nwe P, Bril V. Comparison of IVIg and PLEX in patients with myasthenia gravis. Neurology. 2011;76(23):2017-2023.

15. Zinman L, Ng E, Bril V. IV immunoglobulin in patients with myasthenia gravis: a randomized controlled trial. Neurology. 2007;68(11):837-841.

16. Hoffmann S, Kohler S, Ziegler A, Meisel A. Glucocorticoids in myasthenia gravis - if, when, how, and how much? Acta Neurol Scand. 2014;130(4):211-221.

17. Melzer N, Ruck T, Fuhr P, Gold R, Hohlfeld R, Marx A, Melms A, et al. Clinical features, pathogenesis, and treatment of myasthenia gravis: a supplement to the Guidelines of the German Neurological Society. J Neurol. 2016;263(8):1473-1494.

18. Werner JM, Schweinsberg V, Schroeter M, von Reutern B, Malter MP, Schlaak M, Fink GR, et al. Successful treatment of myasthenia gravis following PD-1/CTLA-4 combination checkpoint blockade in a patient with metastatic melanoma. Front Oncol. 2019;9:84.

19. Bolz S, Ramakrishnan T, Fleischer M, Livingstone E, Stolte B, Thimm A, Kizina K, et al. Detect it so you can treat it: A case series and proposed checklist to detect neurotoxicity in checkpoint therapy. eNeurologicalSci. 2021;22:100324. 